\title{
Design and Implementation of MIMO Transmission through Reconfigurable Intelligent Surface
}

\author{
Wankai Tang*, Jun Yan Dai ${ }^{\dagger}$, Ming Zheng Chen ${ }^{\dagger}$, Kai-Kit Wong ${ }^{\ddagger}$, \\ Xiao $\mathrm{Li}^{*}$, Xinsheng Zhao*, Shi Jin*, Qiang Cheng ${ }^{\dagger}$, and Tie Jun Cui ${ }^{\dagger}$ \\ *National Mobile Communications Research Laboratory, Southeast University, Nanjing, China \\ (e-mail: \{tangwk, li_xiao, xszhao, jinshi\}@ seu.edu.cn). \\ ${ }^{\dagger}$ State Key Laboratory of Millimeter Waves, Southeast University, Nanjing, China \\ (e-mail: \{junyandai, mingzhengc, qiangcheng, tjcui\}@ seu.edu.cn). \\ $\ddagger$ Department of Electronic and Electrical Engineering, University College London, United Kingdom \\ (e-mail: kai-kit.wong@ucl.ac.uk).
}

\begin{abstract}
By the ability to periodically change the reflection coefficients of the unit cells of the reconfigurable intelligent surface (RIS), RIS-empowered wireless transmitter has emerged as an attractive paradigm that can achieve information modulation and transmission in a cost-effective and energy-efficient manner. However, only the phase electromagnetic response of the RISs can be flexibly manipulated in most cases, which significantly limits the transmission rate of RIS-based transmitters. In this paper, RIS-based quadrature amplitude modulation (QAM) and multiple-input multiple-output (MIMO) transmission are designed to improve the communication rate of RIS-based transmitter. In addition, our proposed method of achieving RISbased MIMO-QAM is proved to be robust with the discrete phase shift of the RIS. Furthermore, a prototype is implemented to perform real-time RIS-based MIMO-QAM transmission over the air, which practically validates the proposed approach.
\end{abstract}

\section{INTRODUCTION}

The fifth-generation $(5 \mathrm{G})$ mobile communications is being commercially deployed in many countries around the world. As one of the key technologies of $5 \mathrm{G}$, massive multipleinput multiple-output (MIMO) has become a reality. 5G base stations (BS) are equipped with fully digital massive MIMO radio-frequency (RF) chains and large scale antenna arrays to provide the performance leaps that are being sought in 5G. Meanwhile, the future deployment of millimeter wave (mmWave) technology in 5G will address the spectrum shortage problem in current wireless networks. Nevertheless, the emerging new applications such as virtual reality (VR), augmented reality (AR), holographic projection, autonomous driving, and tactile Internet, to name a few, are leading to the ever-increasing traffic demands that cannot be provided in full scale by $5 \mathrm{G}$ [1]. Therefore, both of the academia and industry begin to discuss and explore the possible enabling technologies of the sixth-generation $(6 \mathrm{G})$ that keeps researchers working in the next decade.

When considering the technologies that may deliver 6G, upgrading massive MIMO to ultra-massive MIMO (UM-MIMO)

This work was supported in part by the National Key Research and Development Program of China 2018YFA0701904, 2018YFA0701602, 2017YFA0700201, 2017YFA0700202, and 2017YFA0700203, the National Science Foundation of China (NSFC) for Distinguished Young Scholars with Grant 61625106, and the NSFC under Grant 61941104, 61722106, and 61731010.
[2] and extending the working spectrum to the terahertz (THz) band [3], appear to be a potential and natural choice. Besides, some potential $6 \mathrm{G}$ enabling technologies, such as large intelligent surface (LIS) [4] and holographic MIMO [5], utilize extremely large antenna aperture to obtain extraordinary channel gain and much improved spatial diversity. However, the tremendously large number of RF chains required by these beyond massive MIMO technologies and the very high working frequency at $\mathrm{THz}$ band will bring about unaffordable hardware cost and energy consumption.

Under this background, reconfigurable intelligent surface (RIS) [6]-[9] emerges as a potential paradigm that can provide a new hardware architecture to alleviate the above mentioned implementation issues. RIS is also known as intelligent reflecting surface (IRS) and passive large intelligent surface (LIS) in the literature, which is often expected to be made of programmable metasurface [10], [11] with electromagnetic (EM) reconfigurable reflecting elements. The EM responses of the reflecting elements (also known as unit cells) of an RIS can be flexibly manipulated through external control signals. The RISs have the ability to engineer the reflected EM waves in real time, which renders them special appealing for wireless communications.

In particular, the RIS-based wireless transmitter can directly modulate the baseband data onto the reflected EM wave, thus provide a cost-effective and energy-efficient approach that does not require conventional RF chains for realizing UMMIMO and related technologies [12], [13]. There have been several prior works on RIS-based transmitter, e.g., [14]-[19]. [14] proposed an RIS-based binary frequency shift-keying (BFSK) transmitter. Subsequently, the experiments of RISbased quadrature phase shift keying (QPSK) transmission were presented in [15] and [16]. An 8-phase shift keying (8PSK) wireless communication prototype empowered by elaborately designed RIS was developed in [17] and [18], respectively. Recently, multi-modulation schemes were realized through an RIS in [19]. However, these prior research efforts were all limited to basic single-input single-output (SISO) communications. Whether RIS-based MIMO transmission is possible is not fully understood, specially when considering the hardware 
constraints of the RIS, such as the discrete phase shift.

In this paper, we study the feasibility of performing MIMO transmission and high-order modulation through RIS-based transmitter. A non-linear modulation technique is introduced to achieve high-order modulation through the RIS. In addition, the impact of the discrete phase shift of the RIS on the system design is analyzed. Further, a prototype is implemented to realize RIS-based MIMO-QAM wireless communication in real time.

\section{SySTEM MODEL}

A general RIS-based MIMO wireless communication system is considered as shown in Fig. 1, in which the reflection coefficient of each unit cell is controlled by a dedicated DAC. The RIS consists of regularly arranged unit cells with $N$ rows and $M$ columns. $U_{n, m}$ denotes the unit cell in the $n^{t h}$ row and $m^{t h}$ column, whose adjustable reflection coefficient is $\Gamma_{n, m}$, for $n \in[1, N]$ and $m \in[1, M]$. The receiver side contains $K$ antennas. $y_{k}$ presents the received baseband signal of the $k^{t h}$ receiving antenna, for $k \in[1, K]$. Let $E_{n, m}, \widetilde{E}_{n, m}, Z_{0}$ and $Z_{n, m}$ represent the EM wave impinging on $U_{n, m}$, the EM wave reflected from $U_{n, m}$, the characteristic impedance of the air, and the equivalent load impedance of $U_{n, m}$, respectively. The reflection coefficient is a physical parameter that describes the complex-valued fraction of the EM wave reflected by an impedance discontinuity in the transmission medium, which can be expressed as

$$
\Gamma_{n, m}=A_{n, m} e^{j \varphi_{n, m}},
$$

where $A_{n, m}$ and $\varphi_{n, m}$ stand for the amplitude alteration and phase shift of the reflected EM wave from $U_{n, m}$, respectively. According to the definition of the reflection coefficient, we have

$$
\widetilde{E}_{n, m}=\Gamma_{n, m} E_{n, m}=A_{n, m} e^{j \varphi_{n, m}} E_{n, m} .
$$

Moreover, the reflection coefficient of $U_{n, m}$ is determined by the characteristic impedance of the air $Z_{0}$ and its equivalent load impedance $Z_{n, m}$, which can be written as [15]

$$
\Gamma_{n, m}=\frac{Z_{n, m}-Z_{0}}{Z_{n, m}+Z_{0}} .
$$

By combining (1) and (3), the amplitude and phase components of $U_{n, m}$ 's reflection coefficient is obtained as

$$
A_{n, m}=\left|\frac{Z_{n, m}-Z_{0}}{Z_{n, m}+Z_{0}}\right|
$$

and

$$
\varphi_{n, m}=\arctan \left(\frac{\operatorname{Im}\left(\frac{Z_{n, m}-Z_{0}}{Z_{n, m}+Z_{0}}\right)}{\operatorname{Re}\left(\frac{Z_{n, m}-Z_{0}}{Z_{n, m}+Z_{0}}\right)}\right) .
$$

Equation (4) and (5) reveal the basic manipulation principle of the reflected EM wave of the RIS: the external control signals adjust the equivalent load impedance $Z_{n, m}$ of each unit cell, thereby regulating the amplitude and the phase of the reflected EM wave in real time.

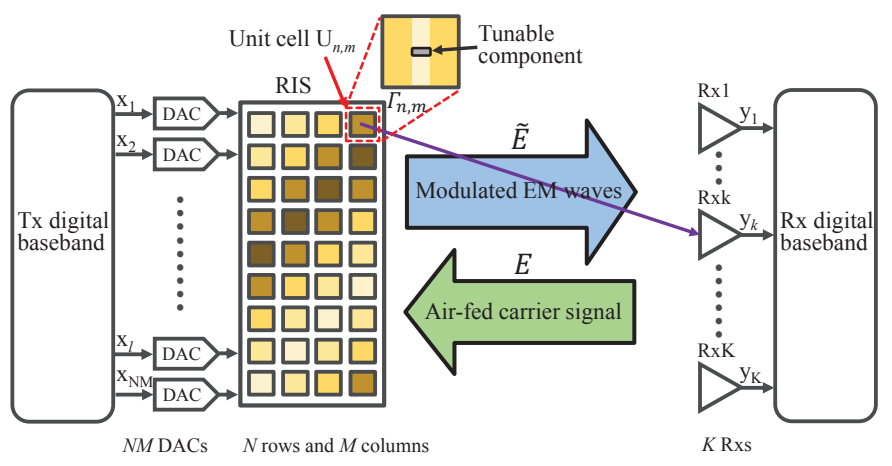

Fig. 1. An RIS-based MIMO wireless communication system.

The EM wave received by the $k^{t h}$ receiving antenna is the superposition of those reflected EM waves by all the unit cells of the RIS, which depends on the wireless channel $h_{n, m}^{k}$ between each unit cell and the $k^{t h}$ receiving antenna, i.e.,

$E_{k}=\sum_{m=1}^{M} \sum_{n=1}^{N} h_{n, m}^{k} \widetilde{E}_{n, m}=\sum_{m=1}^{M} \sum_{n=1}^{N} h_{n, m}^{k} A_{n, m} e^{j \varphi_{n, m}} E_{n, m}$.

Particularly, the incident EM wave $E_{n, m}$ in (6) is a singletone EM wave with frequency $f_{c}$ and acts as the carrier signal, when the RIS is employed as the wireless transmitter. As a result, according to (6) and denoting the noise at the $k^{\text {th }}$ receiving antenna as $n_{k}$, the baseband expression of the received signal of the $k^{t h}$ receiving antenna can be written as

$$
y_{k}=\sum_{m=1}^{M} \sum_{n=1}^{N} h_{n, m}^{k} A_{n, m} e^{j \varphi_{n, m}}+n_{k}=\mathbf{h}_{k} \mathbf{x}+n_{k},
$$

where we denote the channel between the RIS and the $k^{\text {th }}$ receiving antenna as

$$
\mathbf{h}_{k}=\left[h_{1,1}^{k}, h_{1,2}^{k}, \ldots, h_{1, M}^{k}, h_{2,1}^{k}, \ldots, h_{N, M}^{k}\right] \in \mathbb{C}^{1 \times N M},
$$

and the transmitted baseband symbols in vector as

$$
\begin{aligned}
\mathbf{x}= & {\left[x_{1}, x_{2}, \ldots, x_{M}, x_{M+1}, \ldots, x_{N M}\right]^{T} } \\
= & {\left[A_{1,1} e^{j \varphi_{1,1}}, A_{1,2} e^{j \varphi_{1,2}}, \ldots, A_{1, M} e^{j \varphi_{1, M}},\right.} \\
& \left.A_{2,1} e^{j \varphi_{2,1}}, \ldots, A_{N, M} e^{j \varphi_{N, M}}\right]^{T} \in \mathbb{C}^{N M \times 1} .
\end{aligned}
$$

Based on (7), the baseband expression of the RIS-based MIMO wireless communication system is expressed as

$$
\mathbf{y}=\mathbf{H x}+\mathbf{n}
$$

where $\mathbf{y}=\left[y_{1}, \ldots, y_{K}\right]^{T} \in \mathbb{C}^{K \times 1}, \mathbf{H}=\left[\mathbf{h}_{1}, \ldots, \mathbf{h}_{K}\right]^{T} \in$ $\mathbb{C}^{K \times N M}$, and $\mathbf{n} \in \mathbb{C}^{K \times 1}$ are the received signal vector of the receiver as shown in Fig. 1, the wireless channel matrix between the RIS-based transmitter and the receiver, and the noise vector of the receiver, respectively.

Equation (10) reveals that the basic mathematical expression of RIS-based MIMO transmitter is the same as that of the conventional one. Meanwhile, the unique advantage of RIS-based transmitter for being RF chain-free renders it a promising hardware paradigm for new wireless communication technologies, such as UM-MIMO and holographic MIMO that conventional transmitters are hard to realize due to the hardware cost and energy consumption issues. 


\section{Design of RIS-BASED MIMO-QAM TRANSMISSION}

In this section, the design of RIS-based QAM modulation and MIMO transmission is presented. The impact of the discrete phase shift of the RIS on the system design is also discussed and analyzed.

\section{A. RIS-based QAM Modulation}

The prior works on RIS-based transmitter were mainly based on constant envelope modulations, such as QPSK and 8PSK. It is hard for the RIS-based transmitters to perform QAM, because the amplitude and phase responses of each unit cell of the RIS cannot be independently regulated as these two components are usually strongly coupled. In most instances, the electromagnetic responses of the unit cells of the RISs are usually only phase-adjustable. For example, the unit cell in [17] is carefully designed to achieve a continuous phase shift range over $360^{\circ}$, and keep the fluctuation of amplitude response as small as possible. Without loss of generality, we assume that the amplitude response $A_{n, m}=1$ and the phase response $\varphi_{n, m}$ can be flexibly manipulated. Therefore, the baseband expression of RIS-based MIMO described in (10) is converted into a constant envelope MIMO transmission model, in which the transmitted baseband signal is rewritten as

$$
\mathbf{x}=\left[e^{j \varphi_{1,1}}, e^{j \varphi_{1,2}}, \ldots, e^{j \varphi_{1, M}}, \ldots, e^{j \varphi_{N, M}}\right]^{T} .
$$

Here, we introduce a non-linear modulation technique [20] to enable RIS-based QAM modulation under the constant envelope constraint. The baseband symbol of $U_{n, m}$ is defined as

$$
s_{n, m}(t)=\left\{\begin{array}{cl}
e^{j \frac{\Delta \varphi}{T_{s}}\left(t+T_{s}-t_{0}\right)}, & t \in\left[0, t_{0}\right], \\
e^{j \frac{\Delta \varphi}{T_{s}}\left(t-t_{0}\right)}, & t \in\left(t_{0}, T_{s}\right],
\end{array}\right.
$$

in which the phase response of $U_{n, m}$ varies linearly with time in the symbol period $T_{s}, t_{0}$ is the circular time shift, $\frac{\Delta \varphi}{T_{s}}$ characterizes the changing rate of the phase. The noteworthy feature of the baseband symbol described in (12) is that it has two degrees of freedom: $t_{0}$ and $\Delta \varphi$, which enables RIS-based QAM modulation.

Theorem 1. When the baseband symbol is defined as $s(t)=$ $e^{j \frac{\Delta \varphi}{T_{s}}\left(t+T_{s}-t_{0}\right)}$ for $t \in\left[0, t_{0}\right]$, and $s(t)=e^{j \frac{\Delta \varphi}{T_{s}}\left(t-t_{0}\right)}$ for $t \in$ $\left(t_{0}, T_{s}\right]$, the amplitude of its $1^{s t}$ order harmonic component is

$$
\left|a_{1}\right|=\left|\operatorname{sinc}\left(\frac{\Delta \varphi}{2}-\pi\right)\right| \text {, }
$$

and the phase of its $1^{s t}$ order harmonic component is

$$
\begin{aligned}
\angle a_{1}= & -\frac{2 \pi t_{0}}{T_{s}}+\frac{\Delta \varphi}{2}-\pi+\bmod \left(\left\lfloor\frac{\Delta \varphi}{2 \pi}-1\right\rfloor, 2\right) \cdot \pi \\
& +\varepsilon(2 \pi-\Delta \varphi) \cdot \pi,
\end{aligned}
$$

where $\operatorname{sinc}(\cdot), \bmod (\cdot),\lfloor\cdot\rfloor$, and $\varepsilon(\cdot)$ represent sinc function, modulus function, round-down function, and step function, respectively.

Proof: The proof is obtained through exponential Fourier series expansion; details are omitted for space limitations.

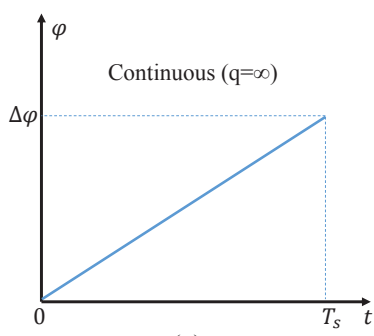

(a)

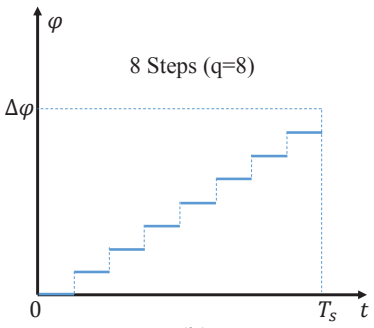

(b)
Fig. 2. Different discrete phase shift steps in the proposed RIS-based QAM symbol. (a) $q=\infty$. (b) $q=8$.

Theorem 1 indicates that RIS-based QAM can be realized on the $1^{s t}$ order harmonic, where $f=f_{c}+\frac{1}{T_{s}}$. In particular, the amplitude modulation and phase modulation are achieved by adjusting $\Delta \varphi$ and $t_{0}$, respectively and independently. As the modulation is carried out on the harmonic, rather than the carrier frequency, we refer to this modulation method as a kind of non-linear modulation.

\section{B. RIS-based MIMO-QAM Transmission}

Theorem 1 provides a non-linear modulation technique for achieving QAM under the constant envelope constraint of the RIS, which makes RIS-based MIMO-QAM transmission possible. By replacing $\mathbf{x}$ in (10) with $\mathbf{s}$, the baseband expression of RIS-based MIMO-QAM wireless communication system is obtained as

$$
\mathbf{y}=\mathbf{H s}+\mathbf{n},
$$

where $\mathbf{s}=\left[s_{1,1}, \ldots, s_{1, M}, \ldots, s_{n, m}, \ldots, s_{N, M}\right]^{T} \in \mathbb{C}^{N M \times 1}$, with $s_{n, m}$ being the transmitted symbol through the unit cell $U_{n, m}$, which has been defined in (12).

On one hand, equation (15) indicates that RIS-based MIMOQAM shares the same mathematical expression with the conventional MIMO-QAM transmitter. Therefore, the widely studied and deployed MIMO communication schemes and algorithms can be applied in the RIS-based MIMO-QAM wireless communication systems. On the other hand, through high-order modulation and MIMO transmission, the proposed RIS-based MIMO-QAM scheme will remarkably improve the transmission rate and spectral efficiency compared to the previous RIS-based transmitter works, meanwhile without any special design requirement on the RIS. In Section IV, we will implement a prototype system to bring the RIS-based MIMOQAM transmission into reality.

\section{Impact of Discrete Phase Shift}

The baseband symbol defined in Theorem 1 for performing RIS-based QAM depends on the assumption that the phase shift of the RIS can continuously change over time as shown in Fig. 2(a). Nevertheless, such an ideal signal does not exist in practical implementation. The phase shift of each unit cell of the RIS is discrete, because its external control signal is generated by the DAC with discrete output characteristic. Denote the number of the discrete phase shift steps as $q$ in a practical baseband symbol of RIS-based QAM. For instance, $q$ is respectively equal to $\infty$ and 8 in Fig. 2(a) and Fig. 2(b). 


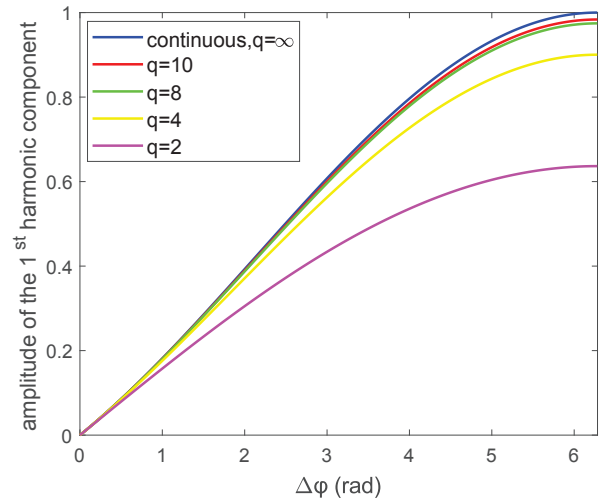

Fig. 3. The amplitude of the $1^{\text {st }}$ order harmonic component with different discrete phase shift steps.

It's important to note that the baseband symbol of RIS-based QAM as ideal as Fig. 2(a) is actually achievable when the unit cell of the RIS is varactor-diode-based type [21] and controlled by the high-resolution DAC (e.g. 16-bit DAC). However, the symbol rate of RIS-based QAM will be significantly limited, since its maximum symbol rate is written as

$$
R_{\text {symbol }}^{\max }=\frac{R_{D A C}}{q},
$$

where $R_{D A C}$ is the maximum sampling rate of the DAC. For example, if $R_{D A C}=100 \mathrm{MSa} / \mathrm{s}$ and $q=1000$, the maximum symbol rate will be limited to a relatively low value of 100 $\mathrm{kS} / \mathrm{s}$. In contrast, a high symbol rate can be obtained when the number of discrete phase shift steps $q$ is small. It is worth noting that the discrete phase constraint here is not the same as that in [9] and [22], in which the RIS is PIN-diode-based type and the phase shift can only take fixed discrete values.

When considering the impact of the discrete phase shift in the system design and letting $t_{0}=0$, the baseband symbol of RIS-based QAM in Theorem 1 is redefined as

$$
\widetilde{s}(t)=e^{j \frac{\Delta \varphi}{q} p}, \text { for } t \in\left[\frac{T s}{q} p, \frac{T s}{q}(p+1)\right),
$$

where $p \in[0,1, \ldots, q-1]$. The $1^{\text {st }}$ order harmonic component of $\widetilde{s}(t)$ is

$$
\begin{aligned}
\widetilde{a_{1}} & =\frac{1}{T_{s}} \int_{0}^{T_{s}} \widetilde{s}(t) e^{-j \frac{2 \pi}{T_{s}} t} d t \\
& =\sum_{p=0}^{q-1} \frac{1}{T_{s}} \int_{\frac{p T s}{q}}^{\frac{(p+1) T s}{q}} e^{j \frac{\Delta \varphi}{q} p} e^{-j \frac{2 \pi}{T_{s}} t} d t \\
& =\frac{\operatorname{sinc}\left(\frac{\pi}{q}\right)}{\operatorname{sinc}\left(\left(\frac{\Delta \varphi}{2}-\pi\right) \frac{1}{q}\right)} \operatorname{sinc}\left(\frac{\Delta \varphi}{2}-\pi\right) e^{j\left(\frac{\Delta \varphi}{2}-\pi-\frac{\Delta \varphi}{2 q}\right) .}
\end{aligned}
$$

Meanwhile, the $1^{\text {st }}$ order harmonic component of the ideal baseband symbol $s(t)$ without discrete phase shift is

$$
a_{1}=\frac{1}{T_{s}} \int_{0}^{T_{s}} e^{j\left(\frac{\Delta \varphi}{T_{s}}-\frac{2 \pi}{T_{s}}\right) t} d t=\operatorname{sinc}\left(\frac{\Delta \varphi}{2}-\pi\right) e^{j\left(\frac{\Delta \varphi}{2}-\pi\right)} .
$$

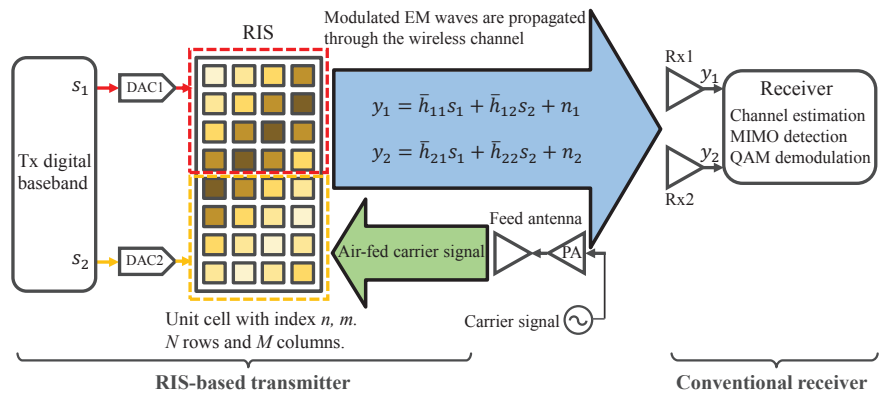

Fig. 4. The diagram of RIS-based $2 \times 2$ MIMO-QAM transmission prototype.

By comparing (18) and (19), we have

$$
\frac{\widetilde{a}_{1}}{a_{1}}=\frac{\operatorname{sinc}\left(\frac{\pi}{q}\right)}{\operatorname{sinc}\left(\left(\frac{\Delta \varphi}{2}-\pi\right) \frac{1}{q}\right)} e^{-j \frac{\Delta \varphi}{2 q}},
$$

which reveals that the larger $q$ will lead to the smaller impact on the baseband symbol of RIS-based QAM. Fig. 3 depicts the amplitude of the $1^{\text {st }}$ order harmonic component under different discrete phase shift steps. It shows that $\left|\widetilde{a}_{1}\right|$ with discrete phase shift steps quickly approaches the ideal $\left|a_{1}\right|$ as $q$ increases. When $q=8$, the impact of the discrete phase shift is already negligible $\left(\frac{\left|\widetilde{a}_{1}\right|}{\left|a_{1}\right|}=0.9745\right.$ when $\left.\Delta \varphi=2 \pi\right)$. Thereby, our proposed approach of achieving RIS-based MIMO-QAM wireless communication is robust with the discrete phase shift, thus a high symbol rate can be achieved.

\section{IMPLEMENTATION OF RIS-BASED MIMO-QAM}

It is obvious that the proposed approach is generalizable for any size of MIMO. Due to the limitation of our experimental hardware condition, an RIS-based $2 \times 2$ MIMO-QAM transmission prototype is implemented here. In spite of that, it is sufficient to show the great potential of realizing UM-MIMO and holographic MIMO technologies through the RISs.

Fig. 4 presents the diagram of the RIS-based $2 \times 2$ MIMOQAM transmission prototype. One bit stream is transmitted by the red half of the RIS and the other bit stream is transmitted by the orange half as shown in Fig. 4. On the basis of the baseband expression of RIS-based MIMO-QAM transmission expressed by (15), we have

$$
\begin{aligned}
& y_{1}=\bar{h}_{11} s_{1}+\bar{h}_{12} s_{2}+n_{1}, \\
& y_{2}=\bar{h}_{21} s_{1}+\bar{h}_{22} s_{2}+n_{2},
\end{aligned}
$$

where $\bar{h}_{11}=\sum_{m=1}^{M} \sum_{n=1}^{\frac{N}{2}} h_{n, m}^{1}, \bar{h}_{12}=\sum_{m=1}^{M} \sum_{n=\frac{N}{2}+1}^{N} h_{n, m}^{1}, \bar{h}_{21}=$ $\sum_{m=1}^{M} \sum_{n=1}^{\frac{N}{2}} h_{n, m}^{2}$, and $\bar{h}_{22}=\sum_{m=1}^{M} \sum_{n=\frac{N}{2}+1}^{N} h_{n, m}^{2}$. Since the signal expression of RIS-based 2x2 MIMO-QAM is the same with that of the conventional MIMO, a common wireless frame structure is designed, which contains one synchronization subframe, one pilot subframe, and sixty data subframes. The pilot subframe comprises 64 RIS-based BPSK symbols and each data subframe consists of 64 RIS-based 16-QAM symbols, which apply QAM modulation on the $1^{\text {st }}$ order harmonic. 


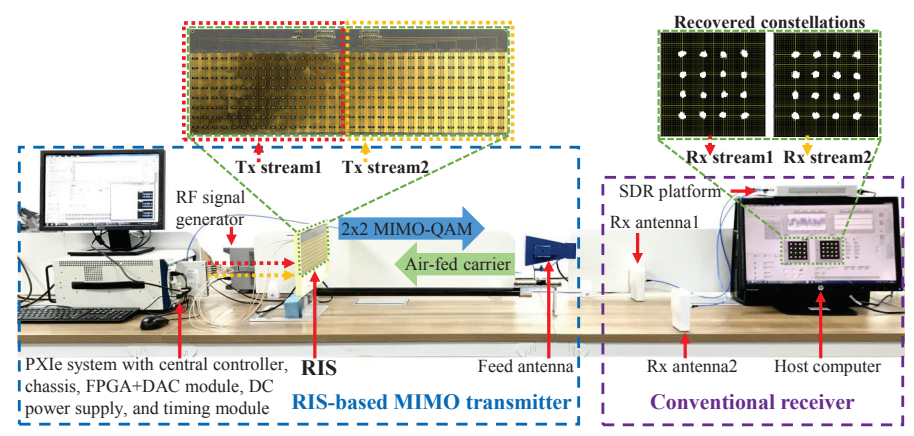

Fig. 5. A photo of the RIS-based $2 \times 2$ MIMO-QAM transmission prototype.

The pilot subframes of these two streams are orthogonal in the time domain, so that the wireless channels $\bar{h}_{11}, \bar{h}_{21}, \bar{h}_{12}$ and $\bar{h}_{22}$ can be easily estimated by the receiver. As shown in Fig. 4, a conventional two-channel receiver is utilized to recover the two transmitted bitstreams, in which least square (LS) and zero forcing (ZF) algorithms are used for channel estimation and channel equalization, respectively.

Fig. 5 illustrates the prototype system, which consists of the RIS, the PXIe system with various peripheral modules, the RF signal generator, the software defined radio (SDR) platform, and the host computer. The RIS here is a kind of varactordiode-based programmable metasurface with 256 unit cells, whose detailed information can be found in [17]. The RIS are controlled by two DAC modules to perform RIS-based $2 \times 2$ MIMO-QAM transmission. The RF signal generator provides the air-fed $4.25 \mathrm{GHz}$ carrier signal through the feed antenna. The SDR platform and host computer form the receiver.

The RIS-based MIMO transmitter is located on the left of Fig. 5, and the receiver is on the right. Real-time RIS-based $2 \times 2$ MIMO-16QAM wireless communication experiment was conducted indoor over the air. The distance between the RIS and the two receiving antennas is about 1.5 meters. The 16QAM constellation diagrams of the two bitstreams recovered by the receiver are clear and dense, which are shown on the upper right hand corner of the figure. When ignoring the overhead of the synchronization and pilot subframes, the data rate of the prototype system reaches $20 \mathrm{Mbps}$ with $2.5 \mathrm{MS} / \mathrm{s}$ symbol rate. The power consumption of the RIS together with its control circuit board is about $0.7 \mathrm{~W}$. As a matter of fact, the transmission data rate can be further improved by increasing the size of MIMO, the modulation order, and the symbol rate in the future.

\section{CONCLUSION}

In this paper, we have presented the approach of achieving QAM modulation and MIMO transmission through the RIS, and proved that the proposed method is robust with the discrete phase shift of the RIS. Furthermore, we have implemented a prototype to realize RIS-based $2 \times 2$ MIMO-16QAM wireless communication with $20 \mathrm{Mbps}$ data rate in real time, which experimentally validates the proposed approach. These encouraging results suggest that RISs provide a promising architecture for realizing UM-MIMO and holographic MIMO technologies, with affordable hardware cost and power consumption.

\section{REFERENCES}

[1] F. Tariq, M. R. A. Khandaker, K.-K. Wong, M. Imran, M. Bennis, and M. Debbah, "A speculative study on 6G," [Online]. Available: https://arxiv.org/abs/1902.06700.

[2] C. Han, J. M. Jornet, and I. F. Akyildiz, "Ultra-massive MIMO channel modeling for graphene-Enabled terahertz-band communications," in Proc. IEEE VTC Spring, Jun. 2018, pp. 1-5.

[3] I. F. Akyildiz, C. Han, and S. Nie, "Combating the distance problem in the millimeter wave and terahertz frequency bands," IEEE Commun. Mag., vol. 56, no. 6, pp. 102-108, Jun. 2018.

[4] S. Hu, F. Rusek, and O. Edfors, "Beyond massive MIMO: The potential of data transmission with large intelligent surfaces," IEEE Trans. Signal Process., vol. 66, no. 10, pp. 2746-2758, May 2018.

[5] C. Huang, S. Hu, G. C. Alexandropoulos, A. Zappone, C. Yuen, R. Zhang, M. Di Renzo, and M. Debbah, "Holographic MIMO surfaces for 6G wireless networks: Opportunities, challenges, and trends," [Online]. Available: https://arxiv.org/abs/1911.12296.

[6] M. Di Renzo et al., "Smart radio environments empowered by reconfigurable AI meta-surfaces: An idea whose time has come," EURASIP J. Wireless Commun. Netw., vol. 2019, no. 1, pp. 1-20, May 2019.

[7] C. Liaskos, S. Nie, A. Tsioliaridou, A. Pitsillides, S. Ioannidis, and I. F. Akyildiz, "A new wireless communication paradigm through softwarecontrolled metasurfaces," IEEE Commun. Mag., vol. 56, no. 9, pp. 162169, Sep. 2018.

[8] Q. Wu and R. Zhang, "Towards smart and reconfigurable environment: Intelligent reflecting surface aided wireless network," IEEE Commun. Mag., vol. 58, no. 1, pp. 106-112, Jan. 2020.

[9] Y. Han, W. Tang, S. Jin, C.-K. Wen, and X. Ma, "Large intelligent surfaceassisted wireless communication exploiting statistical CSI," IEEE Trans. Veh. Technol., vol. 68, no. 8, pp. 8238-8242, Aug. 2019.

[10] T. J. Cui, M. Q. Qi, X. Wan, J. Zhao, and Q. Cheng, "Coding metamaterials, digital metamaterials and programmable metamaterials," Light-Sci. Appl., vol. 3, Oct. 2014.

[11] L. Zhang et al., "Space-time-coding digital metasurfaces," Nat. Commun., vol. 9, Oct. 2018.

[12] W. Tang, J. Y. Dai, M. Chen, X. Li, Q. Cheng, S. Jin, K.-K. Wong, and T. J. Cui, "Subject Editor spotlight on programmable metasurfaces: The future of wireless?," Electron. Lett., vol. 55, no. 7, pp. 360-361, Apr. 2019.

[13] E. Basar, M. Di Renzo, J. De Rosny, M. Debbah, M. Alouini, and R. Zhang, "Wireless communications through reconfigurable intelligent surfaces," IEEE Access, vol. 7, pp. 116753-116773, Aug. 2019.

[14] J. Zhao et al., "Programmable time-domain digital-coding metasurface for non-linear harmonic manipulation and new wireless communication systems," Natl. Sci. Rev., vol. 6, no. 2, pp. 231-238, Mar. 2019.

[15] W. Tang, X. Li, J. Y. Dai, S. Jin, Y. Zeng, Q. Cheng, and T. J. Cui, "Wireless communications with programmable metasurface: Transceiver design and experimental results," China Commun., vol. 16, no. 5, pp. 46-61, May 2019.

[16] J. Y. Dai, W. Tang, J. Zhao, X. Li, Q. Cheng, J. C. Ke, M. Z. Chen, S. Jin, and T. J. Cui, "Wireless communications through a simplified architecture based on time-domain digital coding metasurface," Adv. Mater. Technol., vol. 4, no. 7, pp. 1-8, Jul. 2019.

[17] W. Tang, J. Y. Dai, M. Chen, X. Li, Q. Cheng, S. Jin, K.-K. Wong, and T. J. Cui, "Programmable metasurface-based RF chain-free 8PSK wireless transmitter,' Electron. Lett., vol. 55, no. 7, pp. 417-420, Apr. 2019.

[18] S. Henthorn, K. L. Ford, and T. O. Farrell, "Direct antenna modulation for high-order phase shift keying," IEEE Trans. Antennas Propag., vol. 68, no. 1, pp. 111-120, Jan. 2020.

[19] J. Y. Dai, W. Tang, L. X. Yang, X. Li, M. Z. Chen, J. C. Ke, Q. Cheng, S. Jin, and T. J. Cui, "Realization of multi-modulation schemes for wireless communication by time-domain digital coding metasurface," IEEE Trans. Antennas Propag., vol. 68, no. 3, pp. 1618-1627, Mar. 2020.

[20] J. Y. Dai, J. Zhao, Q. Cheng, and T. J. Cui, "Independent control of harmonic amplitudes and phases via a time-domain digital coding metasurface," Light-Sci. Appl., vol. 3, Nov. 2018.

[21] W. Tang, M. Z. Chen, X. Chen, J. Y. Dai, Y. Han, M. Di Renzo, Y. Zeng, S. Jin, Q., and T. J. Cui, "Wireless communications with reconfigurable intelligent surface: Path loss modeling and experimental measurement," [Online].Available: https://arxiv.org/abs/1911.05326.

[22] Q. Wu and R. Zhang, "Beamforming optimization for wireless network aided by intelligent reflecting surface with discrete phase shifts," IEEE Trans. Commun., vol. 68, no. 3, pp. 1838-1851, Mar. 2020. 